\title{
Ratios of SEP/Suprathermal Intensities and Associated CME Speeds
}

\section{S. W. Kahler*}

Air Force Research Laboratory, Space Vehicles Directorate, Kirtland AFB, NM, USA

E-mail: stephen.kahlerdus.af.mil

\section{A. G. Ling}

Atmospheric Environmental Research, Albuquerque, NM, USA

\begin{abstract}
Recently [1] have found correlations between peak intensities Ip of E > $10 \mathrm{MeV}$ SEP events and suprathermal $\mathrm{H}$ and $\mathrm{He}$ ion intensities observed in situ at $1 \mathrm{AU}$ around the onsets of SEP events. The correlations depend on the solar source longitudes of the driver CMEs and the kind of solar wind (SW) (transient or normal) at the SEP event onset. How the 1 AU suprathermals are related to the solar coronal seed population of SEPs is not known. In a previous study [2] with a similar result, the ratio $\mathrm{R}$ of $20-\mathrm{MeV}$ Ip to the $2-\mathrm{MeV} \mathrm{H}$ suprathermal backgrounds were plotted against CME speeds Vcme. In contrast to the usual strong correlations between Ip and Vcme, [2] found the dependence of $\mathrm{R}$ on $\mathrm{Vcme}$ to be very weak, suggesting that the primary factor determining Ip of SEP events may be the source seed population, rather than Vcme. We extend that work $[1,2]$ by plotting both $\mathrm{R}$ and $\log$ Ip against $\log \mathrm{Vcme}$ for various types of $\mathrm{SW}$ and different source longitudes to look for the dependences of $\mathrm{R}$ on $\mathrm{Vcme}$. We confirm the lower correlations found for $\mathrm{R}$ and $\mathrm{Vcme}$, but $\mathrm{Vcme}$ retains its role as an independent factor in SEP production. The better $\mathrm{R}$ correlation using 1.28-2.56 MeV H suggests a more significant role for lower energy 0.16-0.32 $\mathrm{MeV} \mathrm{H}$ as shock seed particles. Conclusions of variations of $\mathrm{R}$ correlations among different $\mathrm{SW}$ types and solar longitudes are limited by the statistics of the SEP events.
\end{abstract}

36th International Cosmic Ray Conference -ICRC2019-

July 24th - August 1st, 2019

Madison, WI, U.S.A.

${ }^{*}$ Speaker. 


\section{Introduction}

\subsection{SEP Event Peak Intensities and Preceding Suprathermal Intensities}

Several recent works have made connections between suprathermal particle intensities observed at $1 \mathrm{AU}$ and peak intensities Ip or fluences of SEP events. In a survey of 90 large SEP events from 1998 to 2005 [3] found that suprathermal Fe number densities were significantly greater before large 12-80 MeV nucleon ${ }^{-1} \mathrm{Fe}$ fluence events than overall. They suggested suprathermal ion intensities as an input to forecasting probabilities of large SEP events. [2,4] found correlations between logs of Ip and their $2-\mathrm{MeV}$ proton background intensities for $9620-\mathrm{MeV}$ gradual SEP events with sources $>\mathrm{W} 40^{\circ}$, consistent with those background protons as possible seed particle populations. Other works $[2,5,6,7]$ have shown that SEP events occurring in transient solar wind (SW) have statistically larger values of Ip than those occurring in fast or slow SW. Despite the difference, [2] found that the correlations between logs of 20-MeV proton events Ip and of their $2-\mathrm{MeV}$ proton backgrounds were strong for all groups of SW types.

We [1] recently correlated $E>10 \mathrm{MeV}$ SEP event peak intensities Ip with $\mathrm{H}$ and $\mathrm{He}$ intensities at each of two suprathermal energies averaged over four different time periods around the SEP onsets observed in the GOES spacecraft during the period 1998 to 2016. The 201 SEP events were sorted into four groups by their associated solar-source longitude ranges and further separated between events in transient and in normal SW. The larger mean Ip in transient SW than in normal $\mathrm{SW}$ found earlier was confirmed. The suprathermals significantly correlated (Pearson CCs $\approx 0.4-$ 0.6) with SEP Ip from the $0^{\circ}$ to $\mathrm{W} 40^{\circ}$ range, but only weakly $(\mathrm{CC} \approx 0.0-0.3)$ with SEP events from the well connected $\mathrm{W} 41^{\circ}$ to $\mathrm{W} 83^{\circ}$ range, shown in Figure 1. Eastern hemisphere SEP intensities were uncorrelated $(\mathrm{CC} \simeq 0.0)$ with suprathermals in normal $\mathrm{SW}$ but well correlated $(\mathrm{CC} \simeq 0.4$ 0.7 ) for those in transient SW, which [1] interpreted with a simple model involving perpendicular shock acceleration of the suprathermals. In situ B field magnitudes showed no correlation with SEP intensities for any longitude range or time scale. We note that [2] and [1] used the SW structure list of [8] to determine the times of transient SW.

[2] looked at log-log correlations (CCs) of 20-MeV Ip with 2-MeV proton backgrounds and with Vcme. For the former, CCs were 0.60 in transient SW and 0.45 in long-lived (slow and fast) SW. For Vcme correlations, the CCs were 0.74 in transient SW and 0.36 in long-lived SW (rhs of Figure 2). To separate the effects of 2-MeV backgrounds from those of the Vcme on SEP Ip values, [2] normalized Ip to the $2-\mathrm{MeV}$ background by forming the ratios $\mathrm{R} 1=(\mathrm{Ip} / \mathrm{bkgd})$ and plotted $\log$ $\mathrm{R} 1$ values against $\log \mathrm{Vcme}$ as shown in the lhs of Figure 2. R1 was only weakly correlated with $\log \mathrm{Vcme}(\mathrm{CC}=0.17$ for transient SW and 0.25 for long-lived SW). Furthermore, logs of the 2$\mathrm{MeV}$ backgrounds were strongly $(\mathrm{CC}=0.73)$ and weakly $(\mathrm{CC}=0.11)$ correlated with $\mathrm{Vcme}$ in transient and long-lived SW, respectively.

[2] did not separate out longitude ranges in their full set of $96>\mathrm{W} 40^{\circ}$ SEP events and associated CMEs. On the other hand, [1] divided their $201 E>10 \mathrm{MeV}$ proton events and associated suprathermal backgrounds into four longitude ranges, but did not consider the speeds Vcme of associated CMEs. The goal of this work is to combine the two previous results by again plotting log $\mathrm{R}=\log (\mathrm{Ip} / \mathrm{bkgd})$ against $\operatorname{logs}$ of Vcme for SEP events grouped into different SW and longitude groups. From [2], we expect those R dependences to be weaker than plots of log Ip versus log $\mathrm{Vcme}$, but variations with SW type and longitude are also sought in this work. 


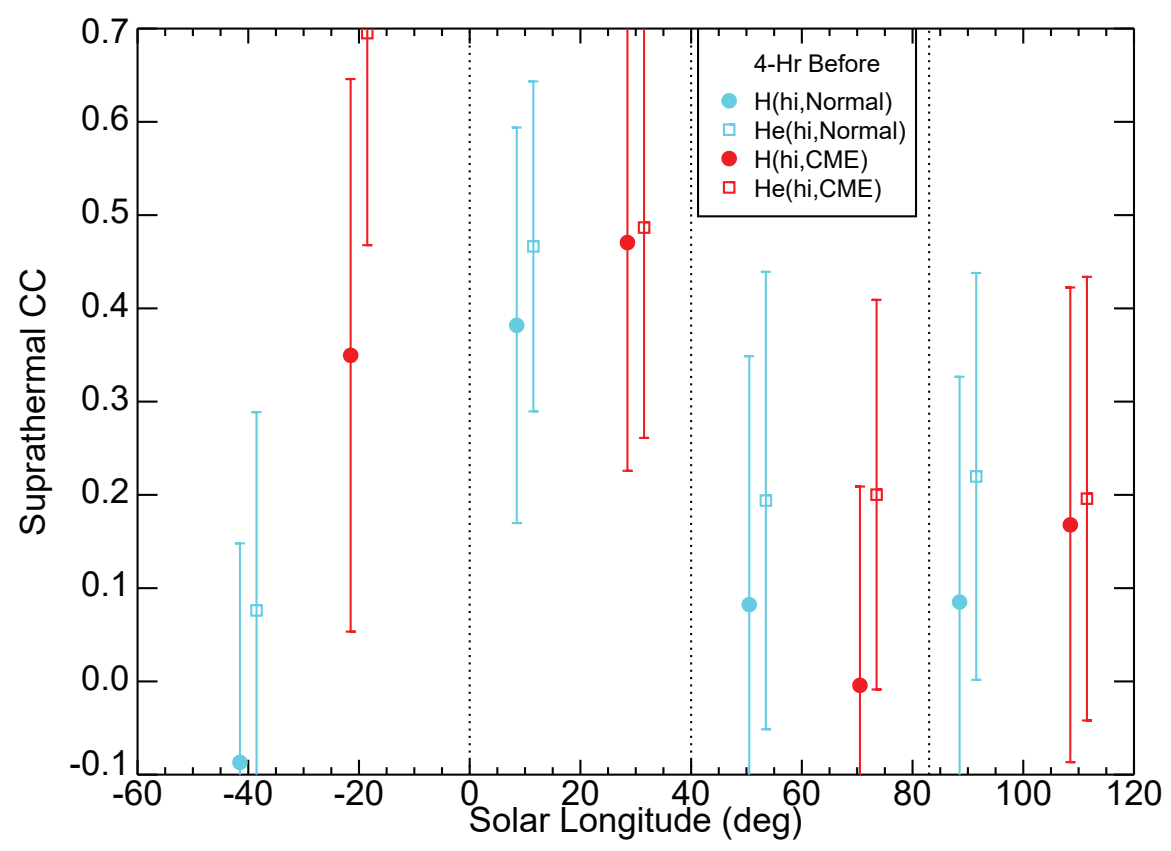

Figure 1: Suprathermal CCs in the 4-hours-before time period for two species, $\mathrm{H}(\mathrm{hi}, 1.28-2.56 \mathrm{MeV})$ and $\mathrm{He}\left(\mathrm{hi}, 1.28-1.81 \mathrm{MeV} \mathrm{nuc}^{-1}\right.$ ), versus solar longitude. Normal and transient (CME) SW period CCs with standard errors are color coded. Vertical dotted lines separate the four longitude ranges. Grey and red symbols show normal and transient SW. From [1].
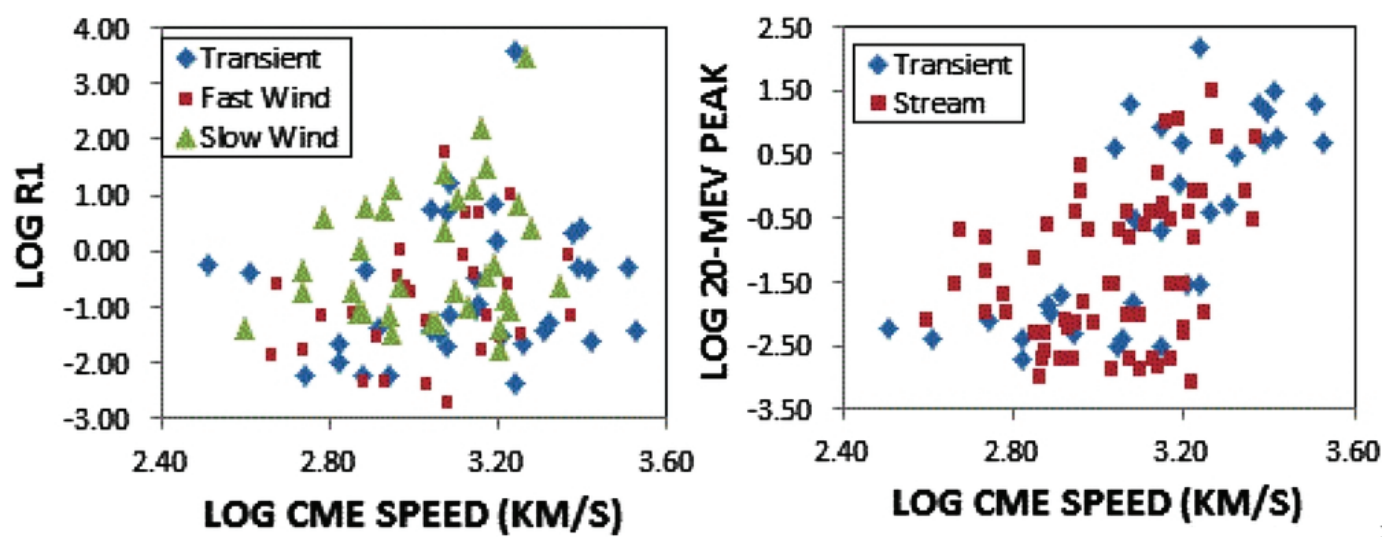

Figure 2: Left: Logs of 20-MeV event peaks Ip normalized to their 2-MeV backgrounds, R1, versus logs of Vcme for three types of SW. The CCs are: transient, 0.17; fast wind, 0.30; and slow wind, 0.24. Right: Logs of Ip versus logs of Vcme. CCs are: transient, 0.74; and stream (fast + slow SW), 0.36. Longitude ranges for events of both panels are $>\mathrm{W} 40^{\circ}$. Combined Figures 2 and 3 of [2]. 


\section{Data Analysis}

We use the list of GOES $E>10 \mathrm{MeV}$ proton events of peak $\geq 1.1$ proton flux units (pfu) in Table 1 of [1], now updated with 6 additional events through the end of 2017, for a total of 207. We report three corrections to their Table 1: 2002 April 21, change Ip from 350 to $3500 \mathrm{pfu} ; 2013$ January 17, change N20E87 to S32W87; and 2013 November 19, change S70W14 to S13W69. For each of the 207 SEP events we sought CME associations at the LASCO CDAW web site and listed their linear speeds Vcme and widths W. Ten events (5 in 1998) were precluded due to LASCO data gaps, leaving 197 SEP events with associated CMEs, of which 77 occurred in transient SW (Richardson, priv. comm.). The event number distribution by longitude bins is: East Hemisphere (43), $0^{\circ}-\mathrm{W} 40^{\circ}(52), \mathrm{W} 41^{\circ}-\mathrm{W} 83^{\circ}(49)$, and $>\mathrm{W} 83^{\circ}$ (53).

For the suprathermal intensities [1] used two elements ( $\mathrm{H}$ and He), two energy ranges $(\sim 0.15$ and 1.5 MeV/nuc, designated lo and hi), and four different time periods around SEP onsets for their extensive Table 2 correlations with SEP Ip. [1] found significant differences among longitudinal ranges but not between the suprathermal elements, energy ranges, or time periods, which tended to track each other in given longitudinal ranges. We select the two suprathermal $\mathrm{H}$ (lo and hi) intensities at the ACE/ULEIS 0.16-0.32 MeV and 1.28-2.56 MeV energy channels, and the 4-hour periods before SEP onsets for our comparisons here. We continue to distinguish between transient and normal SW at the times of SEP onsets.

We form two ratios of proton intensities, $\mathrm{R} 1=\mathrm{Ip} /(0.16-0.32 \mathrm{MeV})$ and $\mathrm{R} 2=\mathrm{Ip} /(1.28-2.56$ $\mathrm{MeV}$ ), to compare with Vcme, similar to the format of Figure 2. For each of the four longitude ranges we correlate the log-log plots of R1, R2 and Ip versus Vcme. The Pearson correlations (CC) are done separately for all, normal and transient SW types of each longitude range. Figures 3 and 4 show examples of all SEP events in the $0-\mathrm{W} 40^{\circ}$ range and of normal SW SEP events in the W41 ${ }^{\circ}$ to $\mathrm{W} 83^{\circ}$ range. The CCs and numbers of SEP events in each correlation are shown in Table 1. We address several questions, the first of which is how much of log-log correlation between Ip and $\mathrm{Vcme}$ is removed when we plot R1 and R2 against Vcme? If the SEP background intensity is the main factor determining Ip, and shock speed (Vcme) is only of secondary importance, then the R1 and R2 correlations with Vcme should be greatly reduced from those of the Ip correlations. The second is how the R1 and R2 correlations vary with solar longitude range and between normal and transient SW, given the variations of Ip versus suprathermals found by [1] in Figure 1. We also ask whether there are any trends that differentiate between the two suprathermal intensities used in R1 and R2. In some cases there were no data for the full 4-hour intervals of the $0.16-0.32 \mathrm{MeV}$ or 1.28-2.56 MeV ULEIS channels, so those events are excluded in the particular set of correlations.

\section{Results}

As in [1], we use the CCs only as a guide to results, owing to the small numbers of cases in most correlations. Taking the correlation probability $p<0.02$ as the requirement for a definitive result, CCs $\geq 0.33,0.42$, and 0.52 are required for $\mathrm{n}=50,30$, and 20 correlated events. The first result is that the R1 correlations (0.12-0.31) with Vcme are lower than those of R2 (0.22-0.57) for all events and for 7 of the 8 separate SW and longitude groups. Remembering that we are testing the Ip to suprathermal intensity ratios versus Vcme, smaller R1 correlations with Vcme should indicate 
4-hour averages before SEP onset 0-40W All SW
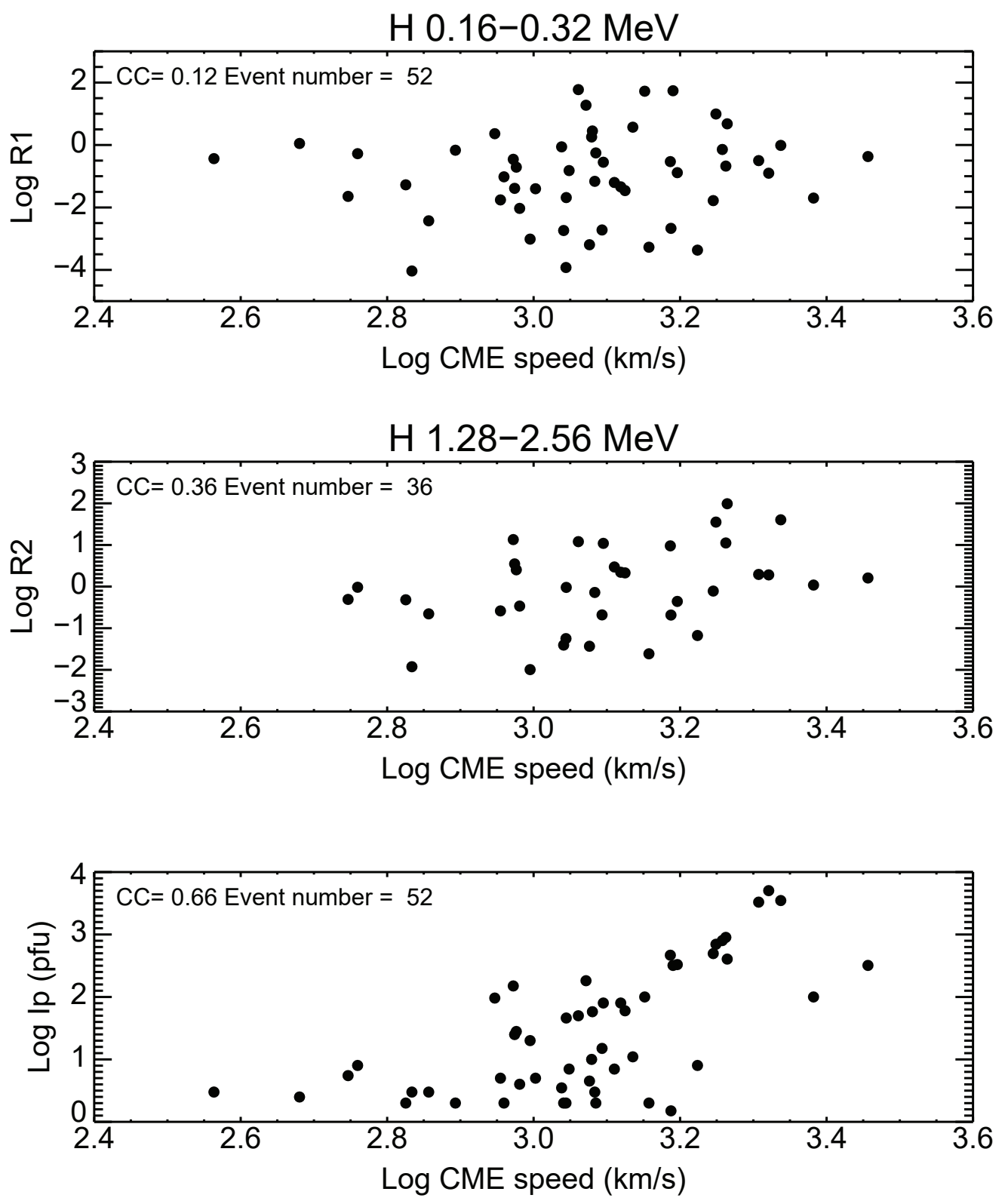

Figure 3: Top: Plot of $\log \mathrm{R} 1$ versus $\log \mathrm{CME}$ speed Vcme for all SEP events in the $0-\mathrm{W} 40^{\circ}$ longitude range. Middle: Same for $\log$ R2. The captions above each panel show suprathermal energy range for each set of R values. Bottom: Log Ip versus log Vcme. Each panel gives the CC values and number of event data points. 


\section{4-hour averages before SEP onset 41W-83W Normal SW}
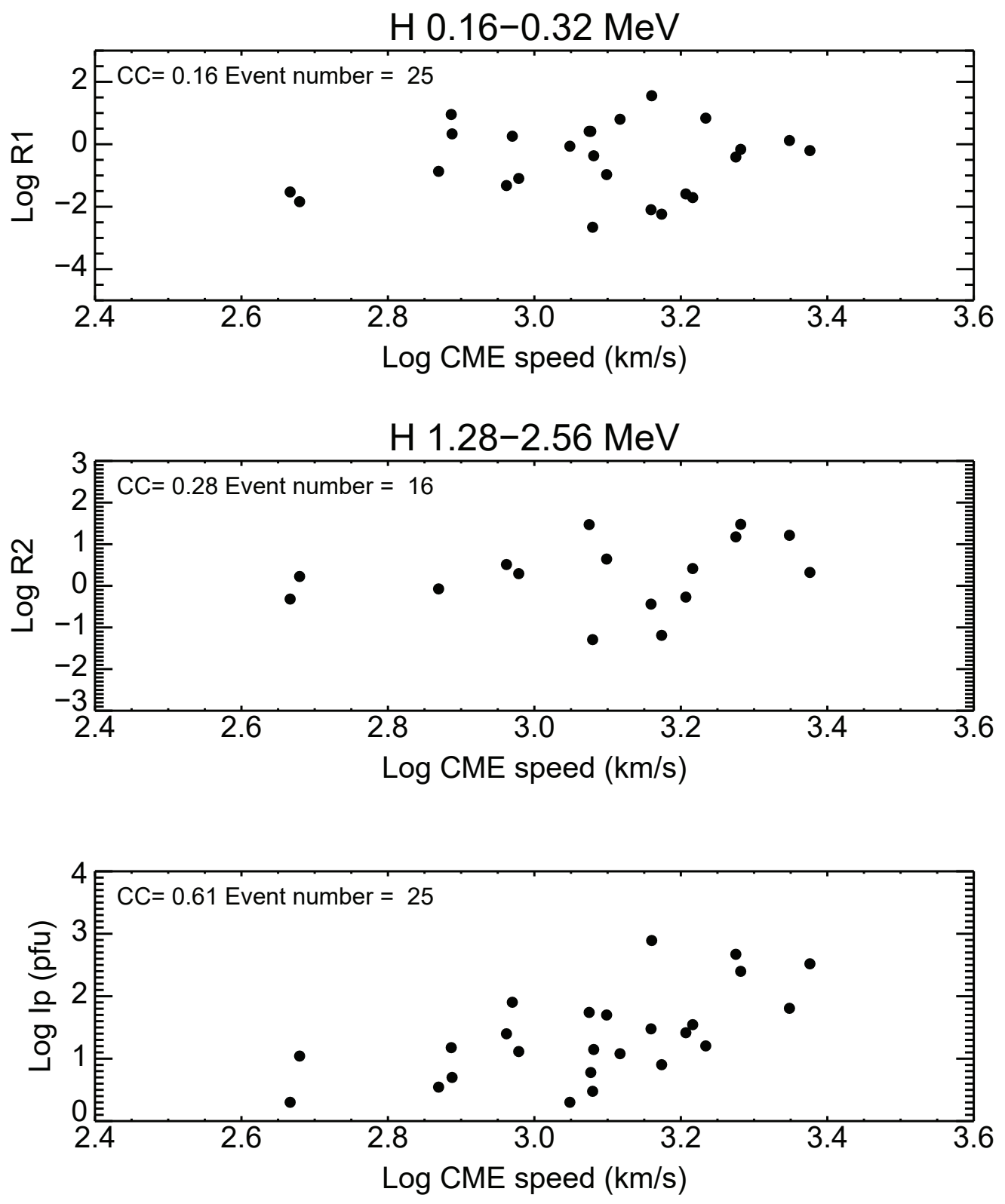

Figure 4: Top: Plot of $\log \mathrm{R} 1$ versus $\log \mathrm{CME}$ speed Vcme for SEP events in the $\mathrm{W} 41^{\circ}-\mathrm{W} 83^{\circ}$ longitude range and normal SW. Middle: Same for log R2. The captions above each panel show suprathermal energy range for each set of R values. Bottom: Log Ip versus log Vcme. Each panel gives the CC values and number of event data points. 
Table 1: Correlation Coefficients (CCs) and Event Numbers (n) for SEP Parameters Vs Logs Vcme

\begin{tabular}{lccccc}
\hline SW Type & Parameter & EL-0 & $0-$ W40 & W41-W83 & $>$ W83 \\
\hline All & Log R1 & $0.31(43)$ & $0.12(52)$ & $0.16(49)$ & $0.17(51)$ \\
& Log R2 & $0.57(29)$ & $0.36(36)$ & $0.22(39)$ & $0.35(35)$ \\
\multirow{4}{*}{ Normal } & Log Ip & $0.52(43)$ & $0.66(52)$ & $0.49(49)$ & $0.58(53)$ \\
& Log R1 & $0.19(28)$ & $0.16(33)$ & $0.16(25)$ & $0.27(32)$ \\
& Log R2 & $0.52(17)$ & $0.34(21)$ & $0.28(16)$ & $0.28(19)$ \\
Transient & Log Ip & $0.31(28)$ & $0.62(33)$ & $0.61(25)$ & $0.35(34)$ \\
& Log R1 & $0.52(15)$ & $0.17(19)$ & $0.38(24)$ & $0.18(19)$ \\
& Log R2 & $0.56(12)$ & $0.46(15)$ & $0.24(23)$ & $0.43(16)$ \\
& Log Ip & $0.70(15)$ & $0.68(19)$ & $0.36(24)$ & $0.57(19)$ \\
\hline
\end{tabular}

a stronger role for the lower energy $0.16-0.32 \mathrm{MeV}$ than the $1.28-2.56 \mathrm{MeV} \mathrm{H}$ suprathermals in the SEP acceleration process. We also get the result of [2] that the ratios R1 and R2 are generally less well correlated with Vcme than are the peak intensities Ip (Figure 2), indicating that Vcme retains an important role in SEP acceleration, as expected for the basic shock-driving concept of fast CMEs.

The high CCs of the 0 -W $40^{\circ}$ suprathermals of Figure 1 suggest that their associated R1 and R2 correlations with Vcme should be substantially reduced from those of Ip with Vcme. Considering only R1, which has lower CCs with Vcme and better statistics (n) than R2, this is clearly the case for CCs of all (0.12 vs 0.66$)$, normal (0.16 vs 0.62$)$ and transient ( 0.17 vs 0.68$)$ SW types. In the $\mathrm{W} 41^{\circ}-\mathrm{W} 83^{\circ}$ range, the $\mathrm{R} 1 \mathrm{CCs}$ are more diminished in the normal $(0.16 \mathrm{vs} 0.61)$ than in the transient ( 0.38 vs 0.36$) \mathrm{SW}$, although from Figure 1, we might expect comparable results. Again contrary to expectations from Figure 1, in the $>\mathrm{W} 83^{\circ}$ range the $\mathrm{R} 1 \mathrm{CC}$ is more reduced in the transient ( 0.18 vs 0.57$)$ than in the normal $(0.27$ vs 0.35$) \mathrm{SW}$ types. In the last longitude range of EL -0, Figure 1 is also not a good guide to the similar reductions of CCs in normal (0.19 vs 0.31 ) and transient ( 0.52 vs 0.70$) \mathrm{SW}$.

The parsing of these correlations to determine the relative importance of the suprathermals and CME speeds to SEP production is difficult because of the correlations between suprathermals and $\mathrm{Vcme}$, as shown in [2]. Thus, a ratio $\mathrm{R}=\mathrm{Ip} /$ suprathermal will scale as $\mathrm{Ip} / \mathrm{Vcme}$ and will be diminished in any plot versus increasing Vcme. In their data sample of $96 \mathrm{SEP}$ events, which was restricted to longitudes $>\mathrm{W} 40^{\circ}$, [2] found a high $(\mathrm{CC}=0.73)$ correlation between the 2$\mathrm{MeV}$ suprathermals and $\mathrm{Vcme}$ in transient $\mathrm{SW}$, but only $\mathrm{CC}=0.11$ for suprathermals and $\mathrm{Vcme}$ in normal SW. That implies that R1 should be much less correlated with Vcme in transient than in normal SW, but that is not obvious in the results in Table 1, for either all longitude ranges or only those $>\mathrm{W} 40^{\circ}$.

\section{Summary}

We have extended the work of [1], who examined variations among the solar source longitudes and SW types in the correlations between intensities Ip of peak $E>10 \mathrm{MeV}$ SEP events and selected suprathermal species measured in situ at $1 \mathrm{AU}$. The diffuse acceleration process for SEP 
events requires both a seed particle population and a CME-driven shock. Correlations of peak SEP event intensities Ip with associated CME speeds Vcme have long been established, so the question now is how the two factors of in situ suprathermal intensities and CME speeds Vcme contribute to the SEP production process. An additional challenge is the correlation between 2$\mathrm{MeV}$ background suprathermals and Vcme found by [2]. We have formed ratios of $E>10 \mathrm{MeV}$ peak proton intensities to two preceding suprathermal populations, R1 and R2, and correlated those ratios to Vcme and then compared those results to Ip correlations with Vcme (Figures 3 and 4). We find, as expected, that the $\mathrm{CCs}$ of ratios $\mathrm{R} 1$ and $\mathrm{R} 2$ versus $\mathrm{Vcme}$ are smaller than those of Ip versus $\mathrm{Vcme}$. When ratios $\mathrm{R}$ themselves correlate with $\mathrm{Vcme}$ [2], the interpretation is difficult. We find that $\mathrm{R} 1$ correlates less well than $\mathrm{R} 2$ with $\mathrm{V} c \mathrm{cme}$, suggesting that the $\mathrm{R} 1$ suprathermal population of $0.16-0.32 \mathrm{MeV} \mathrm{H}$ is a better characteristic of the shock seed population than the $1.28-2.56 \mathrm{MeV} \mathrm{H}$ population. However, the implications of various solar longitudinal and SW type variations of the correlations between Ip and suprathermals found in [1] are not so obvious, partially due perhaps to the sparse statistics.

Acknowledgements. S.K. was funded by AFOSR Task 18RVCOR122. A.L. was supported by AFRL contract FA9453-15-C-0050. CME data were taken from the CDAW catalog. This CME catalog is generated and maintained at the CDAW Data Center by NASA and The Catholic University of America in cooperation with the Naval Research Laboratory. SOHO is a project of international cooperation between ESA and NASA.

\section{References}

[1] S. W. Kahler and A. G. Ling, Suprathermal Ion Backgrounds of Solar Energetic Particle Events, Astrophys.J., 872, (2019), 89.

[2] S. W. Kahler and A. Vourlidas, Solar Energetic Particle Events in Different Types of Solar Wind, Astrophys. J., 791, (2014), 4.

[3] R. A. Mewaldt, G. M. Mason, and C. M. S. Cohen, The Dependence of Solar Energetic Particle Fluences on Suprathermal Seed-Particle Densities, Space Weather: The Space Radiation Environment, AIP Conf. Proc. 1500 (2012), 128.

[4] S. W. Kahler and A. Vourlidas, Do Interacting Coronal Mass Ejections Play a Role in Solar Energetic Particle Events? Astrophys. J., 784, (2014), 47.

[5] S. W. Kahler, A. J. Tylka, and D. V. Reames, A Comparison of Elemental Abundance Ratios in SEP Events in Fast and Slow Solar Wind Regions, Astrophys. J., 701, (2009), 561.

[6] S. W. Kahler, C. N. Arge, S. Akiyama, and N. Gopalswamy, Do Solar Coronal Holes Affect the Properties of Solar Energetic Particle Events?, Solar Phys., 289, (2014), 657.

[7] D. Lario and A. Karelitz, Influence of interplanetary coronal mass ejections on the peak intensity of solar energetic particle events, J. Geophys. Res.: Space Phys., 119, (2014), 4185.

[8] I. G. Richardson and H. V. Cane, Near-earth solar wind flows and related geomagnetic activity during more than four solar cycles (1963-2011), J. Space Weather Space Clim., 2, (2012), A02. 